\section{A transição de saúde e as mudanças na expectativa de vida saudável da população idosa: possíveis impactos da prevenção de doenças crônicas}

\author{
The health transition and changes in healthy life \\ expectancy in the elderly population: possible \\ impacts of chronic disease prevention
}

La transición de la salud y los cambios en la expectativa de vida saludable de la población anciana: posibles impactos de la prevención de enfermedades crónicas

\section{Abstract}

${ }^{1}$ Faculdade de Saúde Pública, Universidade de São Paulo, São Paulo, Brasil. 2 Departamento de Saúde da Coletividade, Faculdade de Medicina do ABC, Santo André, Brasil.

3 Faculdade de Medicina de Ribeirão Preto, Universidade de São Paulo, Ribeirão Preto, Brasil.

Correspondência A. G. Campolina Departamento de Epidemiologia, Faculdade de Saúde Pública, Universidade de São Paulo

Av. Dr. Arnaldo 715, São Paulo SP 01246-904, Brasil.

alecampolina@gmail.com
This study evaluated the impact of the elimination of certain chronic illnesses on disability-free life expectancy in the elderly population. This was a cross-sectional survey based on official data from the city of São Paulo, Brazil, in 2000, and from the SABE study. Cause-deleted probabilities of dying were derived with the cause-elimination life-table technique. Eliminated diseases that generated the largest increase in disability-free life expectancy in women were heart disease, diabetes mellitus, and hypertension (in that order). In men, eliminated diseases that generated the largest increase in disability-free life expectancy were, at 60 years, heart disease, hypertension, and falls, and at 75 years of age, heart disease, hypertension, and chronic lung disease. Classification of chronic diseases according to impact on disability-free life expectancy can assist the planning of preventive programs and health promotion.

Health Transition; Chronic Disease; Active Life Expectancy; Aged
Alessandro Gonçalves Campolina 1

Fernando Adami 2

Jair Licio Ferreira Santos 3

Maria Lúcia Lebrão 1

\section{Resumo}

O objetivo foi avaliar o impacto da eliminação das doenças crônicas sobre a expectativa de vida livre de incapacidade da população idosa. Estudo transversal, de base populacional, utilizando dados oficiais secundários para o Município de São Paulo, Brasil, em 2000, e dados obtidos do Estudo SABE. Tábuas de vida de eliminação de causas foram utilizadas para calcular as probabilidades de morte com a eliminação de doenças. As enfermidades eliminadas que geraram o maior aumento de expectativa de vida livre de incapacidade, para o sexo feminino, foram a doença cardíaca (em primeiro lugar), o diabetes mellitus (em segundo lugar) e a hipertensão arterial (em terceiro lugar). Já para o sexo masculino, as doenças eliminadas que geraram maior aumento de expectativa de vida livre de incapacidade foram a doença cardíaca (em primeiro lugar), a hipertensão arterial (em segundo lugar), a queda (em terceiro lugar aos 60 anos) e a doença pulmonar crônica (em terceiro lugar aos 75 anos). A classificação das doenças crônicas, segundo o impacto na expectativa de vida livre de incapacidade, poderá auxiliar no planejamento de programas de prevenção e promoção da saúde.

Transição Epidemiológica; Doenças Crônicas; Expectativa de Vida Ativa; Idoso 


\section{Introdução}

O aumento na prevalência de doenças crônicas não transmissíveis (DCNT) é uma das principais características do processo de transição epidemiológica, ocorrido primeiramente nos países desenvolvidos e que vem ocorrendo de maneira rápida no Brasil a partir da década de 19601.

A prevalência de algumas dessas doenças eleva-se a partir dos 60 anos, destacando-se: as doenças osteoarticulares, a hipertensão arterial sistêmica (HAS), as doenças cardiovasculares, o diabetes mellitus, as doenças respiratórias crônicas, a doença cerebrovascular e o câncer ${ }^{2}$. Dados nacionais apontam que as DCNT respondem por $66,3 \%$ da carga de doença, enquanto as doenças infecciosas, por $23,5 \%$, e causas externas, por $10,2 \% 3$.

O impacto das DCNT na saúde populacional pode ser avaliado com a utilização de medidasresumo, que estimam a contribuição específica de determinadas condições na expectativa de vida saudável. Dessa forma, indicadores como a expectativa de vida livre de incapacidade podem ser empregados para estimar os ganhos esperados com a redução ou eliminação dessas doenças 4 . Essas abordagens permitem que os benefícios de intervenções possam ser estimados para utilização em análises de custo-efetividade, contribuem para o planejamento de políticas públicas e de programas em saúde, além de fornecerem informações sobre necessidades de pesquisa e desenvolvimento 4 .

Estudos realizados em outros países mostram que, em diferentes situações, a eliminação de doenças crônicas poderia levar a ganhos em expectativa de vida livre da incapacidade $5 . \mathrm{Na}$ Austrália, por exemplo, a eliminação das doenças circulatórias na população idosa levaria aos maiores ganhos de anos de vida saudável, tanto em homens como em mulheres; em seguida viriam a eliminação das neoplasias no sexo masculino e a das doenças musculoesqueléticas no sexo feminino 6,7. Na Holanda, a eliminação da doença cardíaca, das artrites e das lombalgias levaria aos maiores ganhos em expectativa de vida livre de incapacidade na população geral ${ }^{8}$. Nos Estados Unidos, a eliminação da mortalidade por doença cardíaca resultaria nos maiores ganhos na expectativa de vida (três anos para homens e quatro anos para mulheres aos 70 anos); no entanto, a eliminação dessa doença nos indivíduos de idade mais avançada poderia levar à adição de mais anos com incapacidade do que sem incapacidade 9,10 .

No Brasil, estudos têm avaliado a expectativa de vida saudável da população geral e da população de idosos. Em 2005, Romero et al. 11 avalia- ram a expectativa de vida saudável da população brasileira, valendo-se da base de dados do Instituto Brasileiro de Geografia e Estatística (IBGE), utilizando duas abordagens: uma com base na autoavaliação do estado de saúde e outra com base na autoavaliação de limitações em atividades de vida diária. Os autores mostraram que as duas abordagens apresentaram estimativas muito próximas, principalmente nas populações de idades mais avançadas, e que a proporção de anos de vida saudável perdidos é mais importante nas mulheres 11 .

Ainda em 2005, Camargos et al. 12 utilizaram a base de dados de 2000 do estudo Saúde BemEstar e Envelhecimento (Estudo SABE) e, avaliando a expectativa de vida com incapacidade funcional em idosos de São Paulo, mostraram que, apesar de as mulheres idosas paulistanas terem apresentado maior expectativa de vida do que os homens, foi menor a proporção de anos vividos livres de incapacidade funcional.

Em 2009, Camargos et al. 13, utilizando a autopercepção do estado de saúde, com informações advindas da Pesquisa Nacional por Amostra de Domicílios (PNAD) de 2003, calcularam a expectativa de vida saudável para a população brasileira com 60 anos e mais. As estimativas também mostraram que as mulheres vivem mais, porém o número de anos a serem vividos por elas percebendo sua saúde como ruim é maior do que a estimativa para os idosos do sexo masculino.

Assim, considerando que $66,3 \%$ da carga da doença no Brasil decorrem do grande impacto das DCNT e que, em várias regiões do mundo, as análises têm mostrado que a eliminação de doenças crônicas poderia levar a ganhos em expectativa de vida livre da incapacidade, a hipótese central deste estudo é que o mesmo possa ocorrer na população de idosos da comunidade de São Paulo.

Ademais, considerando que outros estudos realizados no contexto brasileiro ainda não avaliaram o efeito da eliminação de doenças crônicas na expectativa de vida livre da incapacidade, o objetivo desta investigação é avaliar o impacto da eliminação das doenças crônicas na expectativa de vida livre de incapacidade da população idosa no Município de São Paulo.

As estimativas obtidas permitirão classificar as diferentes doenças crônicas conforme o impacto na expectativa de vida livre da incapacidade, podendo auxiliar na alocação de recursos e no planejamento da atenção à saúde para a população idosa de São Paulo. 


\section{Métodos}

\section{Delineamento, população e amostragem}

Estudo transversal que se utiliza dos dados coletados pelo Estudo SABE, pesquisa de base populacional cujo objetivo foi avaliar as condições de vida dos idosos de sete cidades de países da América Latina e Caribe (Argentina, Barbados, Brasil, Chile, Cuba, México e Uruguai) 14.

No Brasil, o Estudo SABE foi desenvolvido em 2000 na área urbana do Município de São Paulo. Participaram da pesquisa 2.143 indivíduos de ambos os sexos com idades de 60 anos ou mais. A amostragem foi probabilística e representativa da população idosa do município em 1996, a qual era de 836.223 15. A amostra foi composta, inicialmente, por 1.568 idosos e, posteriormente, ampliada para 2.143 a fim de complementar o número de idosos com idade de 75 anos ou mais e do sexo masculino, em virtude da maior taxa de mortalidade desse grupo etário e sexo 16 .

Para a obtenção da quantidade de idosos desejados, definiu-se o número mínimo de 5.882 domicílios a serem sorteados, pela expressão $[\mathrm{d}=$ $\left.\left(1.500^{*} 10 / 3\right) / 0,85\right]$, sendo d o número mínimo de domicílios a serem sorteados; 1.500, o número mínimo de idosos para possibilitar as análises desejadas; a razão 10/3, referente a três idosos para cada dez domicílios; e 0,85 , a taxa de sucesso esperada para localização e realização das entrevistas nos domicílios sorteados 16 .

Para o sorteio dos domicílios, foi utilizada a amostragem por conglomerados, em dois estágios, selecionados por critério de partilha proporcional ao tamanho:

- Primeiro estágio: foi utilizado como amostra o cadastro permanente de 72 setores censitários, tomados da PNAD de 1995. A fração de amostragem foi calculada por $\mathrm{f}_{1}=\left(72^{*} \mathrm{D}_{\mathrm{i}}\right) / \mathrm{D}$, sendo $\mathrm{f}_{1}$ a fração de amostragem deste estágio; $\mathrm{D}_{\mathrm{i}}$, o número de domicílios em cada setor; e D, o total de domicílios existentes no Município de São Paulo em 1998.

- Segundo estágio: para a amostragem deste estágio, calculou-se o número de domicílios a serem sorteados, segundo o número de setores censitários $(5882 / 72=81,69)$, aproximado para 90. A fração de amostragem do segundo estágio é definida por $\mathrm{f}_{2}=90 / \mathrm{D}_{\mathrm{i}}$, sendo $\mathrm{f}_{2}$ a fração de amostragem deste estágio e $\mathrm{D}_{\mathrm{i}}$, o número de domicílios em cada setor. A atualização dos endereços foi realizada pelas listas da PNAD de 199816.

A probabilidade de um domicílio pertencer à amostra sorteada foi definida por $\mathrm{f}=\mathrm{f} 1 * \mathrm{f} 2$. Nos domicílios sorteados, foram identificados 1.852 idosos elegíveis, dos quais 1.568 concordaram em participar do estudo $(84,67 \%)$.
A complementação da amostra de idosos com idade igual ou superior a 75 anos, do sexo masculino, foi realizada pela localização de moradias próximas aos setores selecionados ou, no máximo, dentro dos limites dos distritos dos setores sorteados 16 .

\section{Coleta de dados}

Os dados coletados foram obtidos por meio de questionário elaborado por um comitê regional, composto por pesquisadores de cada país participante e por especialistas em temas específicos. O instrumento é composto por 11 seções, que abrangem aspectos da vida do idoso: dados pessoais, avaliação cognitiva, estado de saúde, estado funcional, medicamentos, uso e acesso a serviços, rede de apoio familiar e social, história laboral e fontes de ingresso, características da moradia, antropometria, flexibilidade e mobilidade.

As entrevistas foram realizadas em domicílio, sendo a maior parte (88\%) com os idosos. Nos casos em que havia dificuldade ou impossibilidade de o idoso responder (problemas físicos ou cognitivos), solicitou-se a participação de uma pessoa próxima.

A coleta dos dados ocorreu de janeiro de 2000 a março de 2001 em duas etapas: na primeira, foram realizadas as seções de A a J por equipe de 25 profissionais de saúde, incluindo enfermeiras e assistentes sociais, que atuavam individualmente; na segunda etapa, as seções $\mathrm{L}$ e $\mathrm{K}$, que incluem medidas antropométricas e testes de mobilidade e flexibilidade, foram realizadas por equipe de 12 estudantes de nutrição que trabalhavam em dupla .

\section{Variáveis selecionadas}

Foram selecionadas as variáveis referentes às características sociodemográficas, doenças autorreferidas e capacidade funcional. Dentre as sociodemográficas, foram consideradas idade, sexo, arranjo familiar, estado marital, cor da pele, escolaridade e renda, com base em literatura científica pertinente 17,18 .

A idade foi avaliada como variável contínua e categorizada em: 60-74 anos e 75 anos e mais. $\mathrm{O}$ arranjo familiar foi dicotomizado em: vive sozinho(a) ou acompanhado(a). Com relação à escolaridade, os idosos foram categorizados em sem educação formal, ensino fundamental, ensino médio e ensino superior ou mais. O estado marital foi categorizado em solteiro, casado/ amasiado, viúvo e divorciado/separado. O estado laboral foi dicotomizado em trabalha ou não trabalha atualmente. 
As doenças crônicas foram avaliadas com base nas respostas afirmativas (autorreferidas) relativas à presença de nove doenças crônicas: hipertensão arterial sistêmica, diabetes mellitus, doença cardíaca, doença pulmonar, câncer, artropatia, doença cerebrovascular, queda no último ano e problema nervoso ou psiquiátrico. Em virtude da alta prevalência na população idosa e do grande impacto em termos de morbimortalidade, optou-se por tratar o evento queda como doença crônica 19.

A incapacidade funcional foi definida como a dificuldade em realizar uma ou mais atividades de vida diária, dentre as quais foram contempladas: vestir-se, comer, tomar banho, ir ao banheiro, atravessar um cômodo da casa, sofrer de incontinência fecal e de incontinência urinária 20 .

\section{Análise de eliminação de doenças}

Tábuas de vida abreviadas, a partir da idade de 60 anos e para o ano de 2000, foram calculadas com base nas informações de mortalidade e morbidade, utilizando o método de Sullivan 21 e seguindo uma série de etapas, conforme descrito detalhadamente em outra publicação 22

A população idosa estimada para o meio do ano de 2000 foi obtida do Censo Demográfico de 2000, e as informações sobre óbitos na população idosa provieram da Fundação Sistema Estadua de Análise de Dados 23 e DATASUS (Departamento de Informática do SUS. Mortalidade. http:// www.tabnet.datasus.gov.br/cgi/tabcgi.exe?sim/ cnv/obtuf, acessado em 22/Mar/2010).

Para análise do efeito da eliminação de uma doença crônica nas estimativas de expectativa de vida e expectativa de vida livre de incapacidade, foi utilizada abordagem de Nusselder et al. ${ }^{8}$. Nessa proposta, se uma doença é eliminada, os indivíduos não serão acometidos por incapacidade ou óbito decorrente dela. Assumindo a independência entre as causas de óbito e de incapacidade, a eliminação de uma doença levará a um declínio nas probabilidades de morte específicas para a idade e na prevalência de incapacidade específica para a idade.

A análise de regressão logística múltipla foi utilizada para estimar a probabilidade de incapacidade com a eliminação de uma causa (doença crônica), controlada para a idade, sendo, portanto, a presença de incapacidade utilizada como variável dependente ( $1=1$ ou mais incapacidades; 0 = nenhuma incapacidade), enquanto a idade (contínua) e as doenças foram utilizadas como variáveis independentes $(1=$ tem a doença; 0 = não tem)

A probabilidade de uma pessoa ter uma ou mais incapacidades foi computada substituindo- se os coeficientes da regressão e os escores dos respondentes nas variáveis independentes da equação de regressão:

$P=\frac{\varepsilon^{\beta x}}{1+\varepsilon^{\beta x}}$

sendo $\mathrm{P}=$ probabilidade de uma pessoa ter pelo menos uma incapacidade, $\varepsilon=$ base do logaritmo natural e $\beta x=\alpha+\beta_{1} x_{1}+\beta_{2} x_{2}+\ldots$ um vetor de coeficientes de regressão, em que $\mathrm{x}$ são as variáveis.

O efeito da eliminação da doença na prevalência de incapacidade foi simulado eliminandose a doença da equação de regressão obtida. A diferença entre a prevalência total de incapacidade e a prevalência com a eliminação pode, então, ser atribuída à doença eliminada ${ }^{8}$. Em seguida, as probabilidades de morrer, com a eliminação de uma doença, foram estimadas com tábuas de vida de eliminação de causas.

Finalmente, as probabilidades de morte estimadas com eliminação de uma causa específica e a prevalência de incapacidade, estimada com eliminação causal por meio do modelo de regressão logística, foram combinadas em expectativas de vida total e expectativas de vida livre de incapacidade pelo método de Sullivan ${ }^{21}$.

\section{Análise estatística e aspectos éticos}

Foram utilizadas frequências relativas (\%), para a análise das prevalências, e o teste de Rao-Scott, para as associações 24 . Todas as análises, incluindo as regressões logísticas multivariadas, foram realizadas ou no software Microsoft Excel 2007 (Microsoft Corp., Estados Unidos) ou no pacote estatístico Stata, versão 11.1 (Stata Corp., College Station, Estados Unidos), levando em consideração a ponderação da amostra (comando $s v y$ do Stata).

O projeto de pesquisa Estudo SABE foi submetido aos respectivos comitês de ética dos países envolvidos. No Brasil, foi aprovado pelo Comitê de Ética em Pesquisa com Seres Humanos da Faculdade de Saúde Pública (FSP), da Universidade de São Paulo (USP), e pelo Comitê Nacional de Ética em Pesquisa (CONEP).

\section{Resultados}

No ano de 2000, o Estudo SABE avaliou um total de 2.143 idosos, sendo 1.256 pertencentes ao sexo feminino $(58,6 \%)$ e 897 , ao sexo masculino $(41,4 \%)$.

Observou-se maior porcentagem de mulheres $(24,2 \%)$ no grupo etário de idade mais avançada (75 anos e mais), em comparação com os homens (19,2\%). Em relação à cor da pele, as 
distribuições não variaram de modo estatisticamente significativo $(\mathrm{p}=0,9535)$. Quanto à escolaridade, $8,9 \%$ dos homens concluíram o ensino médio, em comparação com $6,8 \%$ das mulheres; $8,3 \%$ dos homens concluíram o ensino superior (ou mais), enquanto $3,7 \%$ das mulheres o fizeram. Observou-se maior porcentagem de viuvez entre as mulheres $(43,2 \%)$, em comparação com os homens $(14,5 \%)$, e maior porcentagem de indivíduos solteiros, também entre as mulheres (52,4\%), quando comparadas aos homens $(4,4 \%)$. Uma maior porcentagem dos homens trabalhava na ocasião do estudo $(40,5 \%)$, diferentemente do percentual feminino $(19,1 \%)$; $17,1 \%$ das mulheres viviam sozinhas, ao passo que $7,7 \%$ dos homens se encontravam nessa condição (Tabela 1).

A prevalência de incapacidade em todas as atividades de vida diária foi, para cada sexo, maior no grupo etário de 75 anos e mais. As atividades de se vestir e incontinência urinária foram as mais prevalentes, independentemente do sexo e da idade.

Observou-se que, para homens e mulheres, as doenças mais prevalentes foram, pela ordem, hipertensão, doença articular, queda e doença cardíaca, com valores maiores nas mulheres (Tabela 2). No sexo feminino, as enfermidades mais incapacitantes foram doença cerebrovascular, mental e articular. Nos homens, tais doenças

Tabela 1

Distribuição dos idosos por sexo, segundo grupo etário, cor da pele, escolaridade, estado marital, estado laboral e arranjo domiciliar. Estudo SABE, Município de São Paulo, Brasil, 2000

\begin{tabular}{|c|c|c|c|}
\hline Variável & Feminino (\%) & Masculino (\%) & Valor de $p$ * \\
\hline \multicolumn{4}{|l|}{ Grupo etário (anos) } \\
\hline $60-74$ & 75,8 & 80,8 & \\
\hline 75 e mais & 24,2 & 19,2 & \\
\hline Total & 100,0 & 100,0 & 0,0030 \\
\hline \multicolumn{4}{|l|}{ Cor da pele } \\
\hline Branca & 76,7 & 76,6 & \\
\hline Parda & 14,0 & 14,2 & \\
\hline Preta & 4,4 & 4,1 & \\
\hline Amarela/Outras & 4,9 & 5,1 & \\
\hline Total & 100,0 & 100,0 & 0,9535 \\
\hline \multicolumn{4}{|l|}{ Escolaridade } \\
\hline Sem educação formal & 23,4 & 18,3 & \\
\hline Ensino Fundamental & 66,1 & 64,5 & \\
\hline Ensino Médio & 6,8 & 8,9 & \\
\hline Ensino Superior ou + & 3,7 & 8,3 & \\
\hline Total & 100,0 & 100,0 & 0,0002 \\
\hline \multicolumn{4}{|l|}{ Estado marital } \\
\hline Solteiro & 5,3 & 4,4 & \\
\hline Casado & 37,5 & 70,4 & \\
\hline Viúvo & 43,2 & 14,5 & \\
\hline Divorciado/Separado & 14,0 & 10,7 & \\
\hline Total & 100,0 & 100,0 & $<0,0000$ \\
\hline \multicolumn{4}{|l|}{ Estado laboral } \\
\hline Trabalha atualmente & 19,1 & 40,5 & \\
\hline Não trabalha atualmente & 80,9 & 59,5 & \\
\hline Total & 100,0 & 100,0 & $<0,0000$ \\
\hline \multicolumn{4}{|l|}{ Arranjo domiciliar } \\
\hline Vive sozinho & 17,1 & 7,7 & \\
\hline Vive acompanhado & 82,9 & 92,3 & \\
\hline Total & 100,0 & 100,0 & $<0,0000$ \\
\hline
\end{tabular}

* Teste de Rao-Scott. 
também se apresentaram como entre as mais incapacitantes, destacando-se a doença cerebrovascular e o diabetes (Tabela 2).

As Tabelas 3 e 4 apresentam: a expectativa de vida, a expectativa de vida livre de incapacidade, a expectativa de vida com incapacidade, a proporção de anos a serem vividos livres de incapacidade conforme a eliminação de doenças crônicas e os rankings correspondentes para as doenças eliminadas, no sexo feminino e masculino, respectivamente.

Na Tabela 3, verifica-se que, em relação ao sexo feminino, as doenças eliminadas que geraram a maior proporção de anos a serem vividos livres de incapacidade foram a doença cardíaca (em primeiro lugar), o diabetes mellitus (em segundo lugar) e a hipertensão arterial (em terceiro lugar). No sexo masculino, as primeiras posições foram ocupadas pela doença cardíaca e pela hipertensão arterial, diferindo apenas no terceiro lugar, no qual observamos a queda, aos 60 anos, e a doença pulmonar crônica, aos 75 anos (Tabela 4).

\section{Discussão}

Conforme os resultados deste estudo, a eliminação de doenças crônicas na população idosa poderia levar a um aumento na expectativa de vida livres de incapacidade em homens e mulheres, tanto aos 60, quanto aos 75 anos. A doença cardíaca apresentou-se como aquela que mais promoveria ganhos em porcentagens de anos a serem vividos livres de incapacidade, caso fosse eliminada, em ambos os sexos. Entre as mulheres, a hipertensão arterial e o diabetes mellitus apareceram em segundo e em terceiro lugares, respectivamente. Já no sexo masculino, a doença cardíaca foi seguida pela hipertensão arterial (segundo lugar), pela queda (terceiro lugar, aos 60 anos) e pela doença pulmonar crônica (terceiro lugar, aos 75 anos)

Entretanto, o contexto em que ocorrem as transformações dos indicadores de saúde de uma população é fundamental para o entendimento das relações entre a expectativa de vida e a expectativa de vida livre de incapacidade 25,26. Assim, é preciso considerar o curso da transição

Tabela 2

Prevalência de doenças crônicas e razão de chances $(R C)$ para a presença de incapacidade em pelo menos uma atividade básica de vida diária, segundo sexo, conforme a análise de regressão logística múltipla. Estudo SABE, Município de São Paulo, Brasil, 2000

\begin{tabular}{|c|c|c|c|c|}
\hline Doenças & Prevalência (\%) & RC & IC95\% & Valor de $p$ \\
\hline \multicolumn{5}{|l|}{ Feminino } \\
\hline Hipertensão arterial sistêmica & 56,60 & 1,47 & $1,09-1,98$ & 0,012 \\
\hline Doença articular & 40,40 & 2,06 & $1,63-2,60$ & $<0,000$ \\
\hline Queda no último ano & 33,00 & 1,50 & $1,15-1,95$ & 0,003 \\
\hline Doença cardíaca & 18,90 & 1,35 & $0,90-2,01$ & 0,138 \\
\hline Diabetes mellitus tipo 2 & 18,70 & 1,44 & $0,97-2,14$ & 0,068 \\
\hline Doença mental & 17,70 & 2,35 & $1,62-3,41$ & $<0,000$ \\
\hline Doença pulmonar crônica & 10,70 & 1,70 & $1,10-2,63$ & 0,019 \\
\hline Doença cerebrovascular & 6,00 & 2,72 & $1,50-4,93$ & 0,001 \\
\hline Neoplasia & 3,50 & 1,59 & $0,61-4,15$ & 0,337 \\
\hline \multicolumn{5}{|l|}{ Masculino } \\
\hline Hipertensão arterial sistêmica & 49,50 & 1,34 & $0,90-2,00$ & 0,143 \\
\hline Doença articular & 20,80 & 1,94 & $1,26-3,01$ & 0,003 \\
\hline Queda no último ano & 22,30 & 1,76 & $1,09-2,83$ & 0,022 \\
\hline Doença cardíaca & 20,50 & 0,86 & $0,53-1,42$ & 0,559 \\
\hline Diabetes mellitus tipo 2 & 17,00 & 2,12 & $1,34-3,35$ & 0,002 \\
\hline Doença mental & 12,50 & 1,90 & $1,03-3,48$ & 0,039 \\
\hline Doença pulmonar crônica & 14,40 & 1,67 & $0,96-2,89$ & 0,067 \\
\hline Doença cerebrovascular & 8,90 & 3,97 & $2,08-7,57$ & $<0,000$ \\
\hline Neoplasia & 3,10 & 1,92 & $0,64-5,78$ & 0,240 \\
\hline
\end{tabular}

IC95\%: intervalo de 95\% de confiança. 
Expectativa de vida, expectativa de vida livre da incapacidade e expectativa de vida com incapacidade, proporção (\%) dos anos a serem vividos livres de incapacidade e rankings correspondentes para doenças eliminadas, no sexo feminino, segundo faixa etária. Município de São Paulo, Brasil, 2000.

\begin{tabular}{|c|c|c|c|c|c|}
\hline Doença & Expectativa de vida & $\begin{array}{l}\text { Expectativa de } \\
\text { vida livre de } \\
\text { incapacidade }\end{array}$ & $\begin{array}{l}\text { Expectativa de vida } \\
\text { com incapacidade }\end{array}$ & $\begin{array}{c}\text { \% expectativa } \\
\text { de vida livre da } \\
\text { incapacidade na } \\
\text { expectativa de vida }\end{array}$ & Posto \\
\hline Mulheres aos 60 anos & 22,45 & 13,01 & 9,44 & 57,94 & \\
\hline Hipertensão arterial sistêmica & 24,60 & 20,91 & 3,69 & 84,99 & 3 \\
\hline Doença articular & 24,45 & 20,10 & 4,35 & 82,21 & 7 \\
\hline Queda no último ano & 24,44 & 20,76 & 3,68 & 84,94 & 4 \\
\hline Doença cardíaca & 25,17 & 21,56 & 3,61 & 85,64 & 1 \\
\hline Diabetes mellitus tipo 2 & 24,71 & 21,04 & 3,67 & 85,14 & 2 \\
\hline Doença mental & 24,49 & 19,86 & 4,63 & 81,09 & 8 \\
\hline Doença pulmonar crônica & 24,61 & 20,62 & 3,99 & 83,80 & 6 \\
\hline Doença cerebrovascular & 24,89 & 19,84 & 5,05 & 79,13 & 9 \\
\hline Neoplasia & 25,49 & 21,47 & 4,02 & 84,23 & 5 \\
\hline Mulheres aos 75 anos & 12,03 & 5,72 & 6,31 & 47,55 & \\
\hline Hipertensão arterial sistêmica & 13,07 & 10,44 & 2,63 & 79,88 & 3 \\
\hline Doença articular & 13,02 & 9,91 & 3,11 & 76,11 & 7 \\
\hline Queda no último ano & 13,02 & 10,39 & 2,63 & 79,77 & 4 \\
\hline Doença cardíaca & 13,23 & 10,70 & 2,53 & 80,87 & 1 \\
\hline Diabetes mellitus tipo 2 & 13,10 & 10,50 & 2,60 & 80,10 & 2 \\
\hline Doença mental & 13,04 & 9,73 & 3,31 & 74,63 & 8 \\
\hline Doença pulmonar crônica & 13,08 & 10,24 & 2,84 & 78,29 & 6 \\
\hline Doença cerebrovascular & 13,18 & 9,61 & 3,57 & 72,92 & 9 \\
\hline Neoplasia & 13,27 & 10,49 & 2,78 & 79,06 & 5 \\
\hline
\end{tabular}

epidemiológica na localidade onde os dados foram obtidos 27 .

Primeiramente, é notório que as doenças cardiovasculares foram e continuam a ser, apesar de sua diminuição, a principal causa de morte no Brasil. Em um estudo sobre o padrão de mortalidade no Município de São Paulo, em 1970, a ordenação dos principais grupos de doenças e agravos à saúde, em função dos ganhos propiciados à expectativa de vida ao nascer, caso não tivessem sido fatores de risco de morte, destacou as doenças cardiovasculares em primeiro lugar 26. A importância dessas condições continua se mantendo ao longo dos anos, em especial nas idades mais avançadas, conforme a avaliação do impacto das causas básicas de morte na expectativa de vida, realizada em Salvador e São Paulo, com base em informações obtidas para o ano de 1996 28,29.

Paralelamente, estudos têm destacado a redução da prevalência das doenças cardiovasculares, de modo que a mortalidade por doença cardíaca isquêmica diminuiu $26 \% 30$. Se a diminuição de prevalência e letalidade dessas condições for levada em conta, essas tendências epidemiológicas podem expressar um efeito positivo na morbidade do idoso, no futuro. Por outro lado, como a prevalência de diabetes e hipertensão arterial no Brasil está aumentando, simultaneamente à prevalência de excesso de peso 30 , dúvidas sobre os impactos da prevenção de doenças cardíacas e cerebrovasculares passam a existir, na medida em que aquelas doenças são fatores de risco conhecidos para estas últimas.

Portanto, a diminuição da prevalência das doenças cardiovasculares e cerebrovasculares, paralelamente ao aumento rápido e continuado da obesidade, hipertensão e diabetes, deixa incertezas sobre a evolução da expectativa de vida livre de incapacidade na população idosa. Seja como for, conforme os resultados deste e de outros estudos, medidas preventivas que enfoquem essas condições, pela sua relevância para esta população, tornam-se um grande desafio 31 .

Em relação ao câncer, estatísticas brasileiras mostram que, nos homens, as taxas de mortalidade por câncer de pulmão, próstata e colorretal estão aumentando; as de câncer gástrico estão 
Expectativa de vida, expectativa de vida livre da incapacidade e expectativa de vida com incapacidade, proporção (\%) dos anos a serem vividos livres de incapacidade e rankings correspondentes para doenças eliminadas, no sexo masculino, segundo faixa etária. Município de São Paulo, Brasil, 2000.

\begin{tabular}{|c|c|c|c|c|c|}
\hline Doença & Expectativa de vida & $\begin{array}{l}\text { Expectativa de } \\
\text { vida livre de } \\
\text { incapacidade }\end{array}$ & $\begin{array}{l}\text { Expectativa de vida } \\
\text { com incapacidade }\end{array}$ & $\begin{array}{c}\% \text { expectativa } \\
\text { de vida livre da } \\
\text { incapacidade na } \\
\text { expectativa de vida }\end{array}$ & Posto \\
\hline Homens aos 60 anos & 17,65 & 13,30 & 4,35 & 75,34 & \\
\hline Hipertensão arterial sistêmica & 24,60 & 20,01 & 4,59 & 81,36 & 2 \\
\hline Doença articular & 24,44 & 19,68 & 4,76 & 80,52 & 6 \\
\hline Queda no último ano & 24,45 & 19,74 & 4,71 & 80,74 & 3 \\
\hline Doença cardíaca & 25,51 & 21,04 & 4,47 & 82,48 & 1 \\
\hline Diabetes mellitus tipo 2 & 24,67 & 19,80 & 4,87 & 80,25 & 7 \\
\hline Doença mental & 24,49 & 19,73 & 4,76 & 80,55 & 5 \\
\hline Doença pulmonar crônica & 24,78 & 20,00 & 4,78 & 80,72 & 4 \\
\hline Doença cerebrovascular & 24,98 & 19,77 & 5,21 & 79,15 & 9 \\
\hline Neoplasia & 25,77 & 20,62 & 5,15 & 80,03 & 8 \\
\hline Homens aos 75 anos & 9,45 & 5,79 & 3,66 & 61,31 & \\
\hline Hipertensão arterial sistêmica & 13,06 & 9,46 & 3,60 & 72,45 & 2 \\
\hline Doença articular & 13,02 & 9,26 & 3,76 & 71,14 & 6 \\
\hline Queda no último ano & 13,02 & 9,31 & 3,71 & 71,47 & 4 \\
\hline Doença cardíaca & 13,23 & 9,85 & 3,38 & 74,43 & 1 \\
\hline Diabetes mellitus tipo 2 & 13,08 & 9,26 & 3,82 & 70,83 & 8 \\
\hline Doença mental & 13,03 & 9,28 & 3,75 & 71,20 & 5 \\
\hline Doença pulmonar crônica & 13,12 & 9,39 & 3,73 & 71,57 & 3 \\
\hline Doença cerebrovascular & 13,16 & 9,12 & 4,04 & 69,35 & 9 \\
\hline Neoplasia & 13,31 & 9,45 & 3,86 & 70,97 & 7 \\
\hline
\end{tabular}

diminuindo e as de câncer de esôfago mantêmse estáveis. Nas mulheres, as taxas de mortalidade por câncer de mama, de pulmão e colorretal aumentaram, enquanto as de câncer do colo do útero e de estômago diminuíram 30. É preciso considerar, ainda, que, no período de 1980 e 2005, a taxa de mortalidade por neoplasias malignas aumentou $25 \%$ na população idosa brasileira 32 .

Diante disso, apesar das dúvidas em relação às tendências da epidemiologia das doenças cardiovasculares, cerebrovasculares e de seus fatores de risco, é possível que as neoplasias ganhem importância, em termos de carga de doença, nas faixas etárias mais avançadas 32,33 .

A doença mental aparece com destaque nos estudos de carga de doença pelo grande impacto em termos de incapacidade e de anos de vida perdidos 8 . No Brasil, séries temporais sobre essas condições não estão disponíveis, mas, no estudo de carga de doença, é notória a participação desse grupo de enfermidades, ocupando a primeira posição no ranking de disability-adjusted life years (DALY) ${ }^{3}$.
No caso específico da população idosa, o aumento progressivo das síndromes demenciais, com o avançar da idade, em especial as do tipo Alzheimer, traz preocupações. Diante da inexistência de medidas de prevenção primária de efetividade comprovada, essas condições possam contribuir para a diminuição da expectativa de vida livre de incapacidade nos idosos 34 .

As síndromes de instabilidade e quedas também apresentam grande relevância para a população idosa ${ }^{35}$. Séries temporais relacionadas a essas síndromes também não estão disponíveis no Brasil, mas, recentemente, o estudo BRAZOS (Brazilian Osteoporosis Study) mostrou que aproximadamente $6 \%$ da população brasileira acima de 40 anos relata ter recebido o diagnóstico médico de osteoporose. Fraturas de baixo impacto foram relatadas por $15,1 \%$ das mulheres e $12,8 \%$ dos homens, confirmando que o relato desta enfermidade pode ter sido subestimado 36. Apesar de ainda não ser possível acompanhar plenamente as tendências de mortalidade e morbidade dessa síndrome 
no contexto brasileiro, o acompanhamento das grandes estratégias preventivas, como o aumento do acesso a programas de manejo de risco destinados à população idosa e da prática da atividade física regular, podem ser sugestivos da boa evolução dessa condição no futuro 37,38 .

Merecem destaque as doenças respiratórias crônicas, que ganham importância nos homens na faixa etária mais avançada; quando eliminadas, são as terceiras a promover a maior proporção de anos a serem vividos livres de incapacidade. Nesse sentido, a queda na taxa de mortalidade ajustada por idade para doenças respiratórias crônicas, que tem sido verificada tanto para DPOC (redução de $28,2 \%$ ), como para asma (redução de $34,1 \%$ ), pode contribuir para uma maior proporção de anos a serem vividos livres de incapacidade no futuro 30 .

As doenças articulares também têm sido destacadas em estudos internacionais, realizados em países desenvolvidos, como importante participante na carga de doença de uma população ${ }^{8}$. A alta prevalência de doença articular autorreferida chama a atenção em outras pesquisas brasileiras que avaliaram a população idosa. No estudo Bambuí, a prevalência de relato de sintomas crônicos nas mãos e joelhos foi de $44,2 \%$ e a de reumatismo diagnosticado por médico foi de $25,3 \%$ (15,3\% nos homens e 31,9\% nas mulheres) 39 . Com o controle das doenças cardiovasculares e das neoplasias, a tendência futura é que tanto essas doenças, como as doenças mentais, ganhem importância progressiva na população idosa.

De maneira geral, o contexto de intensa transição epidemiológica em curso no Brasil ressalta a importância do entendimento sobre as possibilidades de prevenção de doenças crônicas em uma população idosa, numa perspectiva de produção de conhecimentos estratégicos para o planejamento de modelos de atenção adequados a essa população. Nesse sentido, se as políticas de envelhecimento ativo têm por objetivo melhorar a qualidade de vida à medida que as pessoas ficam mais velhas, é necessário, também, reconhecer que "para promover o envelhecimento ativo, os sistemas de saúde necessitam ter uma perspectiva de curso de vida que vise à promoção da saúde, prevenção de doenças e acesso equitativo a cuidado primário e de longo prazo com qualidade" 40 (p. 21).

Diante dessa nova realidade epidemiológica que acompanha o envelhecimento populacional, num contexto em que os custos do setor saúde tornam-se crescentes com a rapidez das inovações tecnológicas, os modelos tecnoassistenciais de gerenciamento de doenças crônicas têm se multiplicado em diversas partes do mundo, com uma necessidade clara de evidências científicas que sustentem uma perspectiva preventiva de atenção à saúde 41 .

Nos Estados Unidos, por exemplo, o Chronic Care Model talvez seja um dos mais conhecidos desses modelos, estando na base da política de Cuidados Inovadores em Doenças Crônicas, proposta pela OMS 42 . Esse modelo busca obter resultados favoráveis no tratamento das condições crônicas de saúde, focando na criação de um ambiente favorável para a relação entre a comunidade, a organização de saúde e os pacientes. Dentre as estratégias preconizadas, figuram a utilização de sistemas informatizados de monitoramento clínico, o treinamento das equipes de saúde com o uso de suportes de decisão, do tipo diretrizes terapêuticas, e a motivação dos pacientes com suporte para o autocuidado 43.

Segundo o relatório da OMS de 2003, os modelos de gerenciamento clínico de doenças crônicas baseiam-se em alguns princípios fundamentais: tomada de decisão com base em evidências científicas, enfoque na população, enfoque na prevenção, enfoque na qualidade, integração entre os níveis de atenção, flexibilidade e adaptabilidade com vigilância permanente ${ }^{44}$. No Brasil, estratégias de saúde que levem em conta esses princípios são de grande importância para a atenção à saúde do idoso e para o manejo das doenças crônicas, no contexto do SUS.

Contudo, a primeira e mais importante limitação do presente estudo é o pressuposto de independência das causas de morte. Sendo assim, estudos de mortalidade por causas múltiplas podem permitir uma melhor avaliação das doenças que contribuem para o óbito por determinada causa, a partir do conhecimento do perfil de morbidade relacionado a doenças subestimadas 45. No Brasil, já existe discussão recente sobre essa abordagem, em estudos realizados com diferentes métodos, como a análise da freqüência de cada diagnóstico como causa básica ou associada de morte 45,46 , a análise de dados multidimensionais por meio da técnica de análise fatorial de correspondência múltipla 47 e o método Grade of Membership (GoM), com base na teoria dos conjuntos nebulosos (“fuzzy sets") 48,49.

A abordagem por causas múltiplas de morte é de grande interesse para o estudo de populações idosas por levar em consideração todas as causas mencionadas na Declaração de Óbito. Torna-se possível, dessa forma, expressar a real magnitude de uma determinada doença como causa básica ou associada de morte 47 ; lidar com a questão da heterogeneidade, comumente encontrada nessas populações 49; e permitir o entendimento de 
como a confluência de doenças compõe mecanismos de causação de mortes 45,48 .

Para a análise de causas múltiplas, os estudos de associações de causas são ainda de uso restrito no contexto brasileiro ${ }^{49}$, porém pesquisas envolvendo as doenças crônicas mais prevalentes na população idosa poderiam trazer grandes contribuições. Outra sinalização importante referese à necessidade de melhoria do preenchimento da Declaração de Óbito, pois a análise de muitas variáveis pode ficar prejudicada pelo alto índice informações ignoradas 47 .

As informações autorreferidas, tanto em relação às doenças crônicas, quanto à presença de incapacidade, também podem trazer vieses aos resultados. No entanto, estudos realizados no Brasil têm evidenciado a validade desse tipo de informação, com boa sensibilidade e especificidade para detectar condições de saúde. As doenças cardiovasculares e o diabetes parecem ser adequadamente reportados pelos indivíduos em virtude da cobertura universal pelo sistema de saúde brasileiro 50 .

Outro aspecto a ser considerado diz respeito à não inclusão de idosos institucionalizados. Isso também poderia gerar uma superestimação do efeito da eliminação de doenças crônicas para essa população em especial, na medida em que esses idosos poderiam estar vivendo em instituições por motivos outros que não doenças crônicas e suas consequências.
Como implicações de pesquisa, considera-se, ainda, que os estudos futuros poderiam utilizar delineamentos longitudinais que permitissem compreender melhor as relações entre as diferentes doenças crônicas e as transições entre os estados de saúde, além de acompanhar as tendências temporais da expectativa de vida livre de incapacidade na população idosa e as diferenças geográficas da expectativa de vida livre de incapacidade, nas várias regiões do país 51,52 . Estudos que incluam a população idosa institucionalizada poderão trazer maiores informações sobre o comportamento de doenças de alta prevalência nestas condições.

Por fim, considerando as características das transições demográfica, epidemiológica e de saúde no Brasil, apesar de o banco de dados utilizado (Estudo SABE) ter sido realizado em 2000, muitas das questões identificadas são ainda presentes em termos de situação de saúde da população idosa. Assim, se o objetivo de uma política de envelhecimento ativo é "aumentar a expectativa de vida saudável, inclusive dos mais frágeis, fisicamente incapacitados e que requerem cuidados" 40 (p. 13), os resultados apresentados neste estudo mostram que a prevenção de doenças crônicas na população idosa é uma possibilidade para a conquista destes objetivos. Além disso, a classificação das doenças crônicas segundo o impacto na expectativa de vida livre de incapacidade poderá auxiliar os tomadores de decisão na alocação eficiente e equitativa de recursos em saúde. 


\section{Resumen}

El objetivo fue evaluar el impacto de la eliminación de las enfermedades crónicas en la expectativa de vida libre de incapacidad de la población anciana. Estudio transversal, de base poblacional, utilizando datos oficiales secundarios sobre el municipio de São Paulo, Brasil, en 2000, y datos obtenidos a partir del Estudio SABE. Las tablas de vida de eliminación de causas se utilizaron para calcular las probabilidades de muerte con la eliminación de enfermedades. Las enfermedades eliminadas que generaron el mayor aumento de expectativa de vida libre de incapacidad, para el sexo femenino, fueron: la enfermedad cardíaca (en lo lugar), la diabetes mellitus (en 2o lugar) y la hipertensión arterial (en 30 lugar). Asimismo, para el sexo masculino, las enfermedades eliminadas que generaron mayor aumento de expectativa de vida libre de incapacidad fueron: la enfermedad cardíaca (en 1o lugar), la hipertensión arterial (en 2o lugar), la caída (en 3o lugar, a los 60 años) y la enfermedad pulmonar crónica (en 3o lugar, a los 75 años). La clasificación de las enfermedades crónicas, según el impacto en la expectativa de vida libre de incapacidad, podrá auxiliar en la planificación de programas de prevención y promoción de la salud.

Transición de la Salud; Enfermedad Crónica;

Esperanza de Vida Activa; Anciano

\section{Colaboradores}

A. G. Campolina, F. Adami, J. L. F. Santos, M. L. Lebrão participaram na concepção, planejamento, análise e interpretação dos dados; participaram na elaboração do rascunho e na revisão crítica do conteúdo e participaram da aprovação da versão final do manuscrito.

\section{Referências}

1. Barreto ML, Teixeira MG, Morais Neto OL, Duarte EC. O nascimento, a vida, o adoecimento, a morte e a atenção à saúde da população brasileira durante 20 anos de Sistema Único de Saúde: uma síntese. In: Ministério da Saúde, organizador. Saúde Brasil 2008: 20 anos de Sistema Único de Saúde (SUS). Brasília: Ministério da Saúde; 2009. p. 407-16.

2. Grundy EMD. The epidemiology of aging. In: Tallis RC, Fillit HW, editors. Brocklehurst's textbook of geriatric medicine and gerontology. Philadelphia: Elsevier Science Ltd.; 2003. p. 3-20.

3. Schramm JMA, Oliveira AF, Leite IC, Valente JG, Gadelha AMJ, Portela MC, et al. Transição epidemiológica e o estudo de carga de doença no Brasil. Ciênc Saúde Coletiva 2004; 9:897-908.
4. Mathers CD, Ezzati M, Lopez AD, Murray CJL, Rodgers A. Causal decomposition of summary measures of population health. In: Murray CJL, Salomon JA, Mathers CD, Lopez AD, editors. Summary measures of population health: concepts, ethics, measurement and applications. Geneva: World Health Organization; 2002. p. 274-90.

5. Perenboom RJM, Van Oyen H, Mutafova M. Health expectancies in European countries. In: Robine JM, Jagger C, Mathers CD, Crimmins EM, Suzman RM, editors. Determining health expectancies. West Sussex: John Wiley \& Sons; 2003. p. 359-76.

6. Mathers CD. Gains in health expectancy from the elimination of diseases among older people. Disabil Rehabil 1999; 21:211-21. 
7. Davis P, Mathers CD, Graham P. Health expectancy in Australia and New Zeland. In: Robine JM, Jagger C, Mathers CD, Crimmins EM, Suzman RM, editors. Determining health expectancies. West Sussex: John Wiley \& Sons; 2003. p. 392-408.

8. Nusselder WJ, van der Velden K, van Sonsbeek JLA, Lenior M, van den Bos GAM. The elimination of selected chronic diseases in a population: the compression and expansion of morbidity. Am J Public Health 1996; 86:187-94.

9. Hayward M, Crimmins E, Saito Y. Cause of death and active life expectancy in the older population of the United States. J Aging Health 1998; 10:192213.

10. Mathers CD. Cause-deleted health expectancies. In: Robine JM, Jagger C, Mathers CD, Crimmins EM, Suzman RM, editors. Determining health expectancies. West Sussex: John Wiley \& Sons; 2003. p. 149-74.

11. Romero DE, Leite IC, Szwarcwald CL. Healthy life expectancy in Brazil: applying the Sullivan method. Cad Saúde Pública 2005; 21 Suppl 1: S7-18.

12. Camargos MCS, Perpetuo IHO, Machado CJ. Expectativa de vida com incapacidade funcional em idosos em São Paulo, Brasil. Rev Panam Salud Pública 2005 ; 17:379-86.

13. Camargos MCS, Rodrigues RN, Machado CJ. Expectativa de vida saudável para idosos brasileiros, 2003. Ciênc Saúde Coletiva 2009; 14:1903-9.

14. Lebrão ML, Laurenti R. Saúde, bem-estar e envelhecimento: o estudo SABE no Município de São Paulo. Rev Bras Epidemiol 2005; 8:127-41.

15. Instituto Brasileiro de Geografia e Estatística. Censo demográfico. Rio de Janeiro: Instituto Brasileiro de Geografia e Estatística; 2001.

16. Silva NN. Aspectos metodológicos: processo de amostragem. In: Lebrão ML, Duarte YAO, organizadores. SABE: saúde, bem-estar e envelhecimento. O projeto SABE no Município de São Paulo: uma abordagem inicial. Brasília: Organização PanAmericana da Saúde; 2003. p. 47-57.

17. Gutiérrez-Fisac JL, Gispert R, Sola J. Factors explaining the geographical differences in disability free life expectancy in Spain. J Epidemiol Community Health 2000; 54:451-5.

18. Mathews RJ, Jagger C, Hancock RM. Does socioeconomic advantage lead to a longer, healthier old age? Soc Sci Med 2006; 62:2489-99.

19. Tinetti ME. Preventing falls in elderly persons. N Engl J Med 2003; 348:42-9.

20. Deeg DJH, Verbrugge LM, Jagger C. Disability measurement. In: Robine JM, Jagger C, Mathers CD Crimins EM, Suzman RM, editors. Determining health expectancies. West Sussex: John Wiley \& Sons; 2003. p. 203-19.

21. Sullivan DF. A single index of mortality and morbidity. HSMHA Health Reports 1971; 86:347-54.

22. Jagger C, Cox B, Le Roy S. Health expectancy calculation by the sullivan method: a practical guide. 3rd Ed. Montpellier: European Health Expectancy Monitoring; 2007. (EHEMU Technical Report 2006, 3).
23. Fundação Sistema Estadual de Análise de Dados Óbitos ocorridos no município de São Paulo, 2000. São Paulo: Fundação Sistema Estadual de Análise de Dados; 2002.

24. Rao JNK, Scott AJ. On chi-squared tests for multiway contingency tables with cell proportions estimated from survey data. Annals of Statistics 1984; 12:46-60.

25. Nusselder WJ. Compression of morbidity. In: Robine JM, Jagger C, Mathers CD, Crimmins EM, Suzman RM, editors. Determining health expectancies. West Sussex: John Wiley \& Sons; 2003. p. 35-58.

26. Johansson SR. The health transition: the cultural inflation of morbidity during the decline of mortality. Health Transit Rev 1991; 1:39-65.

27. Frenk J, Frejka T, Bobadilla JL, Stern C, Lozano R, Sepúlveda J, et al. La transición epidemiológica en América Latina. Bol Oficina Sanit Panam 1991; 111:485-96

28. Gotlieb SLD. Mortalidade diferencial por causa, São Paulo, Brasil, 1970: tábuas de vida de múltiplo decremento. Rev Saúde Pública 1981; 15:401-17.

29. Bardoni AR, Gotlieb SLD. Impacto de causas básicas de morte na esperança de vida em Salvador e São Paulo, 1996. Rev Saúde Pública 2004; 38:16-23.

30. Schmidt MI, Duncan BB, Azevedo e Silva G, Menezes AM, Monteiro CA, Barreto SM, et al. Chronic non-communicable diseases in Brazil: burden and current challenges. Lancet 2011; 377:1949-61.

31. Barreto SM, Pinheiro ARO, Sichieri R, Monteiro CA, Schimidt MI, Lotufo P, et al. Análise da estratégia global para alimentação, atividade física e saúde, da Organização Mundial da Saúde. Epidemiol Serv Saúde 2005; 14:41-68.

32. Lima-Costa MF, Loyola Filho AI, Matos DL. Tendências nas condições de saúde e uso de serviços de saúde entre idosos brasileiros: um estudo baseado na Pesquisa Nacional por Amostra de Domicílios (1998, 2003). Cad Saúde Pública 2007; 23:2467-78.

33. Lima-Costa MF, Matos DL. Tendências das condições de saúde e uso de serviços de saúde da população idosa brasileira: 20 anos de Sistema Único de Saúde. In: Ministério da Saúde, organizador. Saúde Brasil 2008: 20 anos de Sistema Único de Saúde (SUS). Brasília: Ministério da Saúde; 2009. p. 385-405.

34. Machado JCB. Doença de Alzheimer. In: Freitas EV, Py L, Cançado FAX, Doll J, Gorzoni ML, organizadores. Tratado de geriatria e gerontologia. 2a $\mathrm{Ed}$. Rio de Janeiro: Editora Guanabara Koogan; 2006. p. 260-80.

35. Cummings SR, Melton LJ. Epidemiology and outcomes of osteoporotic fractures. Lancet 2002; 359:1761-7.

36. Martini LA, Moura EC, Santos LC, Malta DC, Pinheiro MM. Prevalence of self-reported diagnosis of osteoporosis in Brazil, 2006. Rev Saúde Pública 2009; 43 Suppl 2:1-9.

37. Gregg EW, Pereira MA, Caspersen CJ. Physical activity, falls, and fractures among older adults: a review of the epidemiologic evidence. J Am Geriatr Soc 2000; 48:883-93. 
38. Chang JT, Morton SC, Rubenstein LZ, Mojica WA, Maglione M, Suttorp MJ, et al. Interventions for the prevention of falls in older adults: systematic review and meta-analysis of randomized clinical trials. BMJ 2001; 328:680-6.

39. Machado GPM, Barreto SM, Passos VMA, LimaCosta MF. Projeto Bambuí: prevalência de sintomas articulares crônicos em idosos. Rev Assoc Med Bras (1992) 2004; 50:367-72.

40. Organização Mundial da Saúde. Envelhecimento ativo: uma política de saúde. Brasília: Organização Pan-Americana da Saúde; 2005.

41. Assis M, Hartz ZMA, Valla VV. Programas de promoção da saúde do idoso: uma revisão da literatura científica no período de 1990 a 2002. Ciênc Saúde Coletiva 2004; 9:557-81.

42. Bondenheimer T, Wagner EH, Grumbach K. Improving primary care for patients with chronic illness. JAMA 2002; 288:1775-9.

43. Bondenheimer T, Wagner EH, Grumbach K. Improving primary care for patients with chronic illness: the chronic care model, part 2. JAMA 2002; 288:1909-14.

44. Organização Mundial da Saúde. Cuidados inovadores para condições crônicas: componentes estruturais de ação: relatório mundial. Brasília: Organização Mundial da Saúde; 2003.

45. Furukawa TS, Santo AH, Mathias TAF. Causas múltiplas de morte relacionadas às doenças cerebrovasculares no Estado do Paraná. Rev Bras Epidemiol 2011; 14:231-9.

46. Santo AH. Cysticercosis-related mortality in the State of São Paulo, Brazil, 1985-2004: a study using multiple causes of death. Cad Saúde Pública 2007; 23:2917-27.
47. Rezende EM, Sampaio IBM, Ishitani LH, Martina EF, Vilella LCM. Mortalidade de idosos com desnutrição em Belo Horizonte, Minas Gerais, Brasil: uma análise multidimensional sob o enfoque de causas múltiplas de morte. Cad Saúde Pública 2010; 26:1109-21.

48. Drumond EF, Machado CJ, França E. Óbitos neonatais precoces: análise de causas múltiplas de morte pelo método Grade of Membership. Cad Saúde Pública 2007; 23:157-66.

49. Pereira CCA, Machado CJ, Rodrigues RN. Perfis de causas múltiplas de morte relacionadas ao HIV/ AIDS nos municípios de São Paulo e Santos, Brasil, 2001. Cad Saúde Pública 2007; 23:645-55.

50. Barreto SM, Figueiredo RC. Chronic diseases, selfperceived health status and health risk behaviors: gender differences. Rev Saúde Pública 2009; 43 Suppl 2:S38-47.

51. Cruz GT, Saito Y, Natividad JN. Active life expectancy and functional health transition among Filipino older people. Canadian Studies in Population 2007; 34:29-47.

52. Jagger C, Matthews RJ, Matthews F, Robinson T, Robine J-M, Brayne C. The burden of diseases on disability-free life expectancy in later life. J Gerontol A Biol Sci Med Sci 2007; 62:408-14.

Recebido em 25/Set/2012

Versão final reapresentada em 10/Dez/2012

Aprovado em 31/Jan/2013 